\title{
Tratamiento endoscópico de la obstrucción del tracto de salida gástrico de origen péptico- cicatricial en un niño de 4 años
}

\author{
Endoscopic treatment of cicatricial pyloric stenosis of peptic \\ origin in a 4-year-old boy
}

\author{
Dr. Guillermo R. López Fagalde a, Dr. Leandro N. Manzottia, Dra. Eliana Kellera, \\ Dra. Pilar Hidalgo ${ }^{a}$ Dr. Celso Spomer ${ }^{a}$ y Dr. Gastón Jury ${ }^{a}$
}

\section{RESUMEN}

La obstrucción de la salida gástrica es una afección infrecuente en la edad pediátrica. Tradicionalmente, la cirugía ha sido el modo de tratamiento estándar, pero está asociada a mayor morbimortalidad. El tratamiento endoscópico ha surgido como una alternativa al tratamiento convencional.

Presentamos el caso de un paciente de 4 años con estenosis prepilórica secundaria a enfermedad ulceropéptica. El cuadro clínico empezó con vómitos y dolor abdominal de 2 meses de evolución. Frente a la falta de respuesta al tratamiento médico y a la dilatación endoscópica con balón, se realizaron incisiones radiadas con electrocauterio e inyecciones de esteroides.

La terapéutica endoscópica con balón debería ser el primer gesto terapéutico en este tipo de estenosis refractarias al tratamiento médico.

Palabras clave: estenosis pilórica, obstrucción de la salida gástrica, dilatación, endoscopia.

\section{ABSTRACT}

Gastric outlet obstruction is an uncommon condition in children. Traditionally, surgery has been the standard mode of treatment, but it is associated with higher morbidity and mortality. Endoscopic treatment has emerged as an alternative to conventional treatment. We present the case of a 4-year-old patient with refractory prepyloric stenosis secondary to peptic ulcer disease. The picture begins with vomiting and abdominal pain of 2 months of evolution. Pre-pyloric stenosis was confirmed. Faced with the lack of response to medical treatment and balloon dilation, radiated incisions were made with electrocautery and steroid injections.

When medical treatment is not sufficient, endoscopic balloon therapy should be the first therapeutic gesture in this type of stenosis; given its refractoriness, we believe it is important to highlight the usefulness of endoscopic treatment, which could prevent surgery and associated morbidity and mortality.

Key words: pyloric stenosis, gastric outlet obstruction, dilatation, endoscopy.

Financiamiento:

Ninguno.

Conflicto de intereses: Ninguno que declarar.

Recibido: 22-10-2020

Aceptado: 29-4-2021
Cómo citar: López Fagalde GR, Manzotti LN, Keller E, Hidalgo P, et al. Tratamiento endoscópico de la obstrucción del tracto de salida gástrico de origen péptico-cicatricial en un niño de 4 años. Arch Argent Pediatr 2021;119(5):354-357.

\section{INTRODUCCIÓN}

La obstrucción del tracto de salida gástrica (OTS) en la infancia es una afección infrecuente y tiene una incidencia de 1 en 100000 nacidos vivos cuando se excluye la estenosis hipertrófica del píloro (EHP) del lactante. ${ }^{1}$

La OTS puede deberse a causas congénitas (diafragma antral, atresia pilórica y estenosis hipertrófica del píloro en el lactante) o a causas adquiridas (úlcera péptica, ingestión de cáusticos, tumor, enfermedad granulomatosa crónica y gastroenteritis eosinofílica). ${ }^{2}$

A diferencia de los adultos, ${ }^{3}$ en la población pediátrica la causa más frecuente de las OTS es patología benigna. En el lactante, la EHP como causa congénita y la úlcera péptica (UP) como causa secundaria son las más frecuentes.

El dolor epigástrico, las náuseas y vómitos, la distensión abdominal, la saciedad temprana y la pérdida de peso son los síntomas de presentación más comunes de la OTS.

En general, la evolución de la UP en los niños es más prolongada que en los adultos, por lo que el grado de estenosis puede ser muy significativo. La cicatrización y la remodelación tisular pueden causar OTS en la úlcera péptica crónica. ${ }^{4}$

Tradicionalmente, la cirugía ha sido el modo de tratamiento estándar para la OTS relacionada con la UP (vagotomía con piroloplastia, antrectomía o gastroyeyunostomía y Billroth I, Billroth II o piloroplastia), pero está asociada a mayor morbimortalidad. 
Feng et al., ${ }^{2}$ afirmaron que la OTS benigna puede tratarse de manera eficaz mediante dilatación endoscópica con balón (DEB), para evitar la cirugía.

Se han utilizado técnicas endoscópicas complementarias para aumentar la eficacia de la DEB en las estenosis benignas refractarias, incluidas, entre otras, la inyección de esteroides locales intralesionales como la triamcinolona ${ }^{5,6}$ y la incisión endoscópica con electrocauterización. ${ }^{7,8}$

Se presenta un paciente pediátrico con una estenosis prepilórica secundaria a enfermedad ulceropéptica, sin respuesta al tratamiento médico, que requirió dilataciones progresivas neumáticas y la combinación con otras técnicas endoscópicas, como la inyección de triamcinolona e incisiones con electrocauterio.

\section{CASO CLÍNICO}

Paciente de 4 años, oriundo de la provincia de Entre Ríos, sin antecedentes patológicos de relevancia, que comenzó con vómitos y dolor abdominal, en contexto de ingesta de antiinflamatorios no esteroides (AINE) por cuadro de vías aéreas superiores en los días previos. Los estudios iniciales (incluidas una radiografía simple de abdomen y una ecografía abdominal) descartaron un abdomen agudo quirúrgico. Recibió tratamiento ambulatorio con ranitidina y metoclopramida, presentó una mejoría parcial y transitoria, ya que, a los 10 días, comenzó nuevamente con vómitos diarios y recurrentes de contenido gástrico.

Los estudios de laboratorios generales estaban dentro de los parámetros normales (incluida la serología para celiaquía negativa).

Figura 1. Fotografía obtenida por endoscopia antes del tratamiento

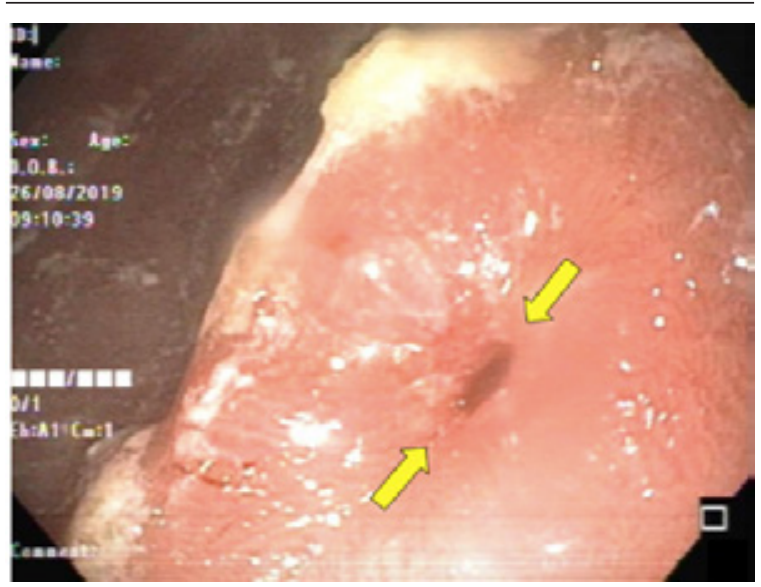

Por el cuadro de vómitos y teniendo en cuenta el antecedente de ingesta de AINE, se solicitó una seriada esófagogastroduodenal, que evidenció el píloro elongado, con una imagen deprimida en la curvatura menor prepilórica. Ante dichos hallazgos, la pérdida de $2 \mathrm{~kg}$ de peso y la falta de resolución del cuadro durante más de 2 semanas a pesar del tratamiento con esomeprazol $10 \mathrm{mg}$ cada 12 horas, fue derivado a nuestro centro para la realización de una videoendoscopia digestiva alta (VEDA) diagnóstica. Este estudio mostró esófago normal, estenosis que impresionaba pilórica, de aspecto inflamatorio, cicatricial, infranqueable al paso del endoscopio pediátrico $(9 \mathrm{~mm}$ de diámetro) (Figura 1) y restos alimenticios en cavidad gástrica. Las muestras de la biopsia de la estenosis evidenciaron componentes inflamatorios y se descartaron gastritis eosinofílica e infección por Helicobater pylori.

Otros estudios realizados: gastrinemia, normal. Tomografía de abdomen con contraste oral que evidenció distensión de cámara gástrica con pasaje filiforme a través del píloro y depresión de la mucosa en ese nivel (Figura 2).

Frente a la falta de respuesta al tratamiento médico, se realizó una VEDA terapéutica con dilatación neumática con balón de expansión radial controlada (tipo CRE, por su sigla en inglés) hasta $10 \mathrm{~mm}$, sin poder franquear la estenosis (Figura 3).

A los 10 días se realizó una segunda dilatación endoscópica hasta $12 \mathrm{~mm}$, se logró franquear la estenosis e identificar el lecho ulceroso prepilórico. Dicha lesión se interpretó como la causante de la fibrosis con retracción del antro prepilórico.

FIGURA 2. Imagen tomográfica que muestra estenosis pilórica

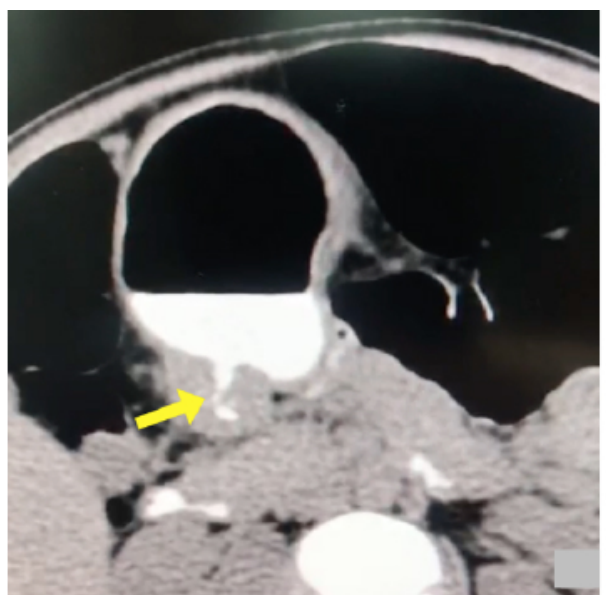


Debido a la persistencia de algunos síntomas, como eructos, vómitos, plenitud posprandial, y a la falta de respuesta sostenida a las dos dilataciones previas, debido al desarrollo de un gran anillo fibrótico en el nivel de la estenosis, se realizó una tercera VEDA con incisiones radiadas con un cuchillo de corte endoscópico (IT-Knife ${ }^{\circledR}$ ) en formas de estrella, principalmente en las zonas con mayor fibrosis, y dilatación con balón de tipo CRE hasta $15 \mathrm{~mm}$ (Figura 4).

El control endoscópico a las 3 semanas evidenció una estenosis de $8 \mathrm{~mm}$ con importante componente fibrótico, motivo por el cual se realizó una cuarta sesión endoscópica, con cortes radiados con IT-Knife ${ }^{\circledR}$ en los sitios con mayor fibrosis, inyección de triamcinolona en el anillo fibrótico y dilatación con balón de tipo CRE hasta $15 \mathrm{~mm}$.

FIGURA 3. Fotografía obtenida por endoscopia durante la segunda sesión, dilatación con balón de tipo CRE

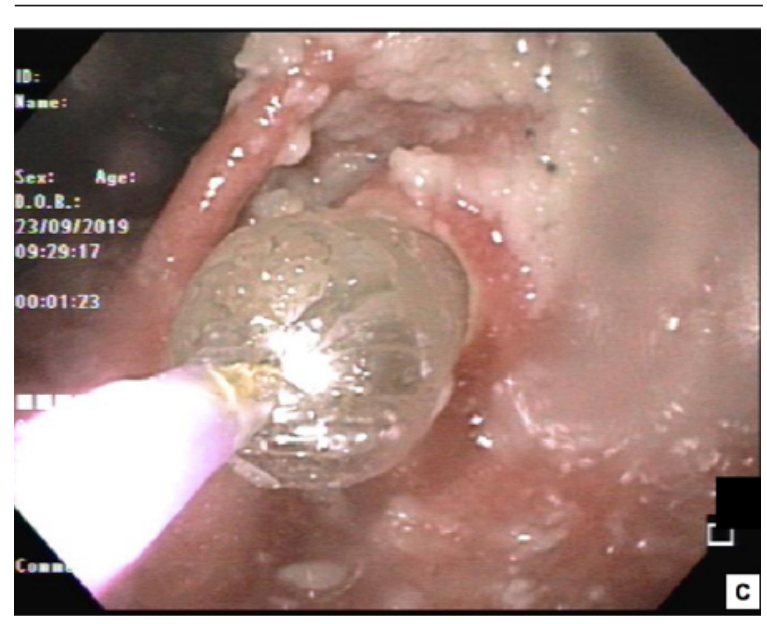

Figura 4. Fotografía obtenida por endoscopia de incisiones radiadas con IT-Knife ${ }^{\circledR}$

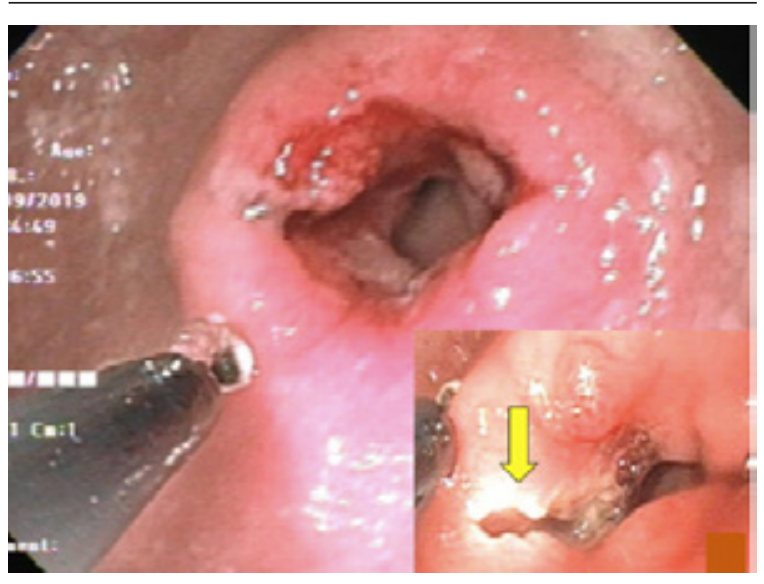

Con la terapéutica realizada, presentó franca mejoría de los síntomas y continuó el tratamiento con esomeprazol $10 \mathrm{mg}$ cada 12 horas durante otras 8 semanas.

El control endoscópico a los 2 meses evidenció píloro permeable con mucosa de aspecto normal (Figura 5).

Todas las dilataciones se realizaron en forma ambulatoria, bajo visión endoscópica y control radiológico, con balones de tipo CRE hasta $15 \mathrm{~mm}$ bajo control manométrico. Las incisiones se realizaron con IT-Knife ${ }^{\circledR}$ en modalidad corte con electrobisturí inteligente (equipo $\mathrm{ERBE}^{\circledR}$ ). Luego de cada procedimiento, el paciente permaneció un promedio de 6 horas en observación, con tolerancia a líquidos previamente al alta.

En total se le realizaron cuatro endoscopias con dilatación; en las dos últimas, se agregaron cortes radiados con IT-Knife ${ }^{\circledR}$ del proceso fibrótico e inyección con triamcinolona en la última sesión.

Al momento de esta publicación, luego de 1 año el paciente se encuentra asintomático.

\section{DISCUSIÓN}

La causa más frecuente de OTS en la edad pediátrica es la estenosis hipertrófica del píloro en el lactante. Las obstrucciones pilóricas secundarias a enfermedad ulcerosa son frecuentes en adultos, aunque excepcionales en pacientes pediátricos. ${ }^{1}$

Tradicionalmente la cirugía fue el tratamiento de elección para la OTS. Muchos estudios indican que la DEB es una alternativa eficaz y segura a la cirugía en pacientes adultos con OTS relacionada con úlcera gástrica. ${ }^{9}$

FIGURA 5. Fotografía obtenida por endoscopia luego del tratamiento

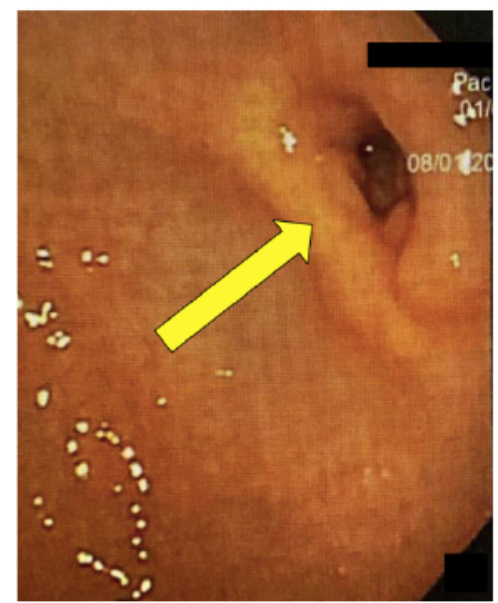


En los pacientes pediátricos, la eficacia y la seguridad dependen de la causa de la OTS. ${ }^{10}$

La mayoría de la bibliografía del uso de DEB para OTS se encuentra en población adulta; hay pocos reportes de esta técnica en pacientes pediátricos.

En los estudios realizados por Sario ${ }^{11}$ sobre DEB en el tratamiento de los niños con estenosis pilórica benigna, las tasas de respuesta variaron entre el $16 \%$ y el $80 \%$.

Hay dos técnicas que pueden mejorar los resultados de las DEB: la inyección de esteroides (triamcinolona) dentro de la lesión y las incisiones con electrocauterización.

Las inyecciones de esteroides intralesionales pueden aumentar el efecto de la DEB, ya que inhiben la formación de estenosis al impedir la síntesis de colágeno, la cicatrización crónica y la fibrosis. ${ }^{4}$

La DEB con incisión endoscópica adicional logró resultados exitosos en la OTS inducida por cáusticos. Boron y cols., ${ }^{12}$ utilizaron con éxito la electrocauterización por vía endoscópica para incidir el segmento estenótico con esfinterótomo en un paciente con estenosis pilórica refractaria.

Luego de descartarse un cuadro de abdomen agudo quirúrgico y aproximadamente tras 1 mes de evolución, el paciente fue derivado a nuestro centro, donde se constató estenosis prepilórica secundaria a una ulcera péptica a través de una endoscopia. Por esto se inició tratamiento endoscópico con dilataciones neumáticas progresivas con balón de tipo CRE. Frente a la respuesta parcial sintomática y reestenosis en los controles endoscópicos, se decidió realizar incisiones radiadas con IT-Knife ${ }^{\circledR}$ utilizando corriente de corte e inyecciones con triamcinolona, dado el gran componente fibrótico de la estenosis. Con esta técnica, se logró una mejoría sintomática inmediata con su correlato endoscópico 2 meses después del procedimiento.

Las incisiones radiadas son una alternativa para el tratamiento de las estenosis refractarias en adultos. Hay poca bibliografía en cuanto a esta técnica en pacientes pediátricos, pero con buenos resultados en combinación con DEB. ${ }^{13,1}$

\section{REFERENCIAS}

1. Sharma KK, Agrawal P, Toshniwal H. Acquired gastric outlet obstruction during infancy and childhood: a report of five unusual cases. J Pediatr Surg. 1997; 32(6):928-30.

2. Feng J, Gu W, Li M, Yuan J, et al. Rare causes of gastric outlet obstruction in children. Pediatr Surg Int. 2005;21(8):635-640.

3. Aljure Reales V, Quintero Moreno C, Salamanca Velandia C, Pérez Ángel N, et al. Estenosis pilórica del adulto, a propósito del primer caso registrado en Colombia. Rev Colomb Radiol. 2016; 27(3):4512-5.

4. Chao HC. Update on endoscopic management of gastric outlet obstruction in children. World J Gastrointest Endosc. 2016; 8(18):635-45.

5. Öztan MO, Güngör-Takeş G, Çağan-Appak Y, Yıldız C, et al. Management of NSAID-related pyloric obstruction in a child using endoscopic balloon dilatation: A case report. Turk J Pediatr. 2018; 60(6):765-8.

6. Thomson M, Tringali A, Dumonceau JM, Tavares M, et al. Paediatric gastrointestinal endoscopy: European Society for Paediatric Gastroenterology Hepatology and Nutrition and European Society of Gastrointestinal Endoscopy Guidelines. J Pediatr Gastroenterol Nutr. 2017; 64(1):133-53.

7. Arango ML, Ricardo AM, Chavarro OO. Incisión radial y corte endoscópico en el manejo de estenosis esofágica anastomótica refractaria. Reporte de un caso. Rev Colomb Gastroenterol. 2018; 33(2):172-5.

8. Lee TG, Yoon SM, Lee SJ. Endoscopic radial incision and cutting technique for treatment-naive stricture of colorectal anastomosis: Two case reports. World J Gastrointest Surg. 2020; 12(11):460-7.

9. Hamzaoui L, Bouassida M,Mansour IB, Medhioub M, et al. Balloon dilatation in patients with gastric outlet obstruction related to peptic ulcer disease. Arab J Gastroenterol. 2015; 16(3-4):121-4.

10. Goenka AS, Dasilva MS, Cleghorn GJ, Patrick MK, et al. Therapeutic upper gastrointestinal endoscopy in children: an audit of 443 procedures and literature review. J Gastroenterol Hepatol. 1993; 8(1):44-51.

11. Khullar SK, DiSario JA. Gastric outlet obstruction. Gastrointest Endosc Clin N Am. 1996; 6(3):585-603.

12. Boron B, Gross KR. Successful dilatation of pyloric stricture resistant to balloon dilatation with electrocautery using a sphinctertome. J Clin Gastroenterol. 1996; 23(3):239-41.

13. Hagiwara A, Sonoyama Y, Togawa T, Yamasaki J, et al. Combined use of electrosurgical incisions and balloon dilatation for the treatment of refractory postoperative pyloric stenosis. Gastrointest Endosc. 2001; 53(4):504-8.

14. Chao HC, Luo CC, Wang CJ. Elimination of postoperative pyloric stricture by endoscopic electrocauterization and balloon dilatation in an infant with congenital antral web. Pediatr Neonatol. 2011; 52(2):106-9. 